\title{
Formation of Faceted Spirals during Directional Eutectic Solidification
}

\author{
Saman Moniri ${ }^{1}$, Hrishikesh Bale ${ }^{2 *}$, Tobias Volkenandt ${ }^{2}$, Stephen Kelly², Ashwin Shahani ${ }^{1}$ \\ 1. University of Michigan, Ann Arbor, MI, United States. \\ 2. Carl Zeiss Microscopy Inc., Pleasanton, CA, United States. \\ * Corresponding author: hrishikesh.bale@zeiss.com
}

Eutectic systems commonly exhibit ordered microstructures that can be broadly classified as lamellar, rod-like, acicular, or globular. Most binary eutectics will display either one or a combination of these morphologies, depending on the material properties and growth conditions. Some ternary eutectic systems have demonstrated the presence of intricate spiraling patterns $[1,2]$. More recently, we have observed exceptionally intricate, faceted spiral eutectic structures upon directional solidification in the $\mathrm{Zn}-\mathrm{Mg}$ alloy system [3, 4]. These two-phase $\mathrm{Zn}-\mathrm{MgZn}_{2}$ microstructures are ordered, metastable, and chiral, opening new opportunities in the field of photonics, where such structures can serve as large area templates for rapid fabrication of complex structures. This promise largely depends on our ability to engineer the microstructure with sufficient degree of control to meet technological demands. The underlying mechanisms that trigger the formation of these spiral microstructures are extremely complex and remain elusive. To better understand the emergence of such structures from a parent liquid phase, a multi-scale, multimodal imaging approach is essential. By combining non-destructive 3D X-ray nano-tomography, 3D electron backscatter diffraction, and high resolution electron microscopy we can gain new insights into the morphology, crystallography, and the driving mechanisms for these structures.

SEM provides sufficient contrast and resolution to discern the morphological details of the spiral architectures (lamellar thicknesses vary between $100 \mathrm{~nm}-1 \mathrm{um}$ ) on the sample surface and provides feedback for the assessment of the synthesis parameters in directional solidification. Detailed morphological selection maps relating the thermal gradient and growth velocity to the obtained eutectic microstructure can be derived. However, eutectic colonies containing clusters of spiral structures are non-uniformly distributed throughout the bulk sample which is not captured by surface imaging techniques. X-ray nano-tomography based on X-ray optics has sufficiently high resolution to reveal the spiral structures and enables inspection of the sub-surface microstructures in 3D non-destructively. The complex morphology of the faceted spiral is likely influenced by the crystallography of the constituent eutectic phases, $\mathrm{Zn}$ and $\mathrm{MgZn}_{2}$. 3D EBSD enables us to locate the nucleation sites of these spirals, identify the crystallographic growth direction, determine the interphase habit plane orientations, and characterize the epitaxial relationships between the two phases across the sample volume. Furthermore, by combining 3D EBSD and high resolution (S)TEM imaging, we can now peer into the very origins of the spiral growth for the first time. Correlative imaging approaches facilitate capturing important features across length scales and help us understand how they are linked hierarchically.

We present here results from the multi-scale investigation of faceted spirals in a binary $\mathrm{Zn}-\mathrm{Mg}$ eutectic alloy obtained using SEM, TEM and X-ray nano-tomography (nTXM). Tying SEM observations with the growth conditions set forth in the directional solidification experiments, we can infer that there is competitive growth between the faceted $\mathrm{Zn}-\mathrm{MgZn}_{2}$ eutectic and the rod-like $\mathrm{Zn}-\mathrm{Mg}_{2} \mathrm{Zn}_{11}$ eutectic. Reconstructed images revealed, for the first time, the 3D morphology of spirals and demonstrated that they form a hexagonal pyramid enveloping shape with an intra-facet angle of $10^{\circ}$ and a dihedral angle of $18^{\circ}$, an observation uniquely made possible through nTXM. It was also observed from the 3D data that 
the direction of the pyramidal axes of the spirals are randomly distributed through the sample volume. 3D-EBSD images provided insights into the epitaxial relationships between the two phases and also clues on why the habit planes meet at a apex. Our correlative imaging workflow provides new insights into the complex morphology, crystallography, and underlying growth mechanism of the faceted eutectic spirals. It is anticipated that these results will provide the necessary benchmark data for simulations (e.g., phase field) of multiphase solidification patterns.

\section{References:}

[1] Witusiewicz, Hecht, Fries, Rex, J. Alloys Compd. 387 (2005), p. 217.

[2] Hötzer, et al., Acta Mater. 106 (2016), p. 249.

[3] Dippenaar, Bridgman, Chadwick, J. Inst. of Metals 99 (1971), p. 137.

[4] Fullman, Wood, Acta Metall. 2 (1954), p. 188.
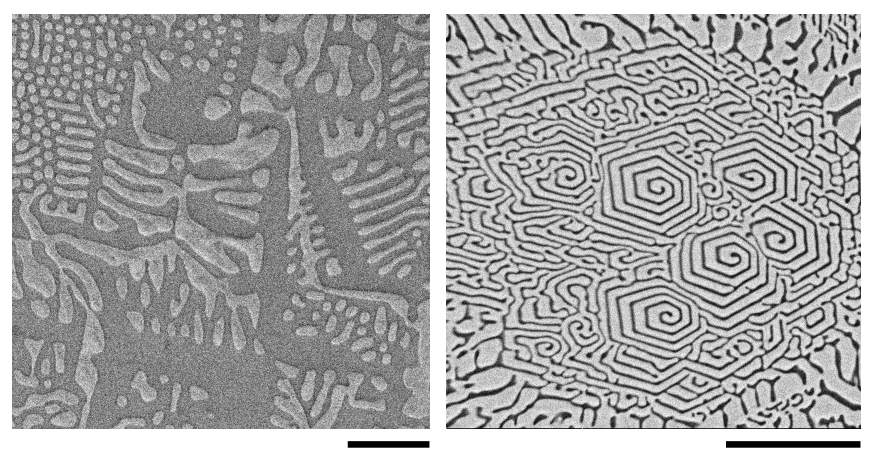

Figure 1. Eutectic morphologies in $\mathrm{Zn}-\mathrm{Mg}$ system. Scanning electron micrographs of the thermodynamically stable, rod-like $\mathrm{Zn}-\mathrm{Mg}_{2} \mathrm{Zn}_{11}$ eutectic (left) and the metastable, faceted spiral $\mathrm{Zn}-\mathrm{MgZn}_{2}$ eutectic (right). In both cases, the light gray phase is $\mathrm{Zn}$, while the dark phase is $\mathrm{Mg}$-rich intermetallic. Scale bars: $20 \mu \mathrm{m}$.
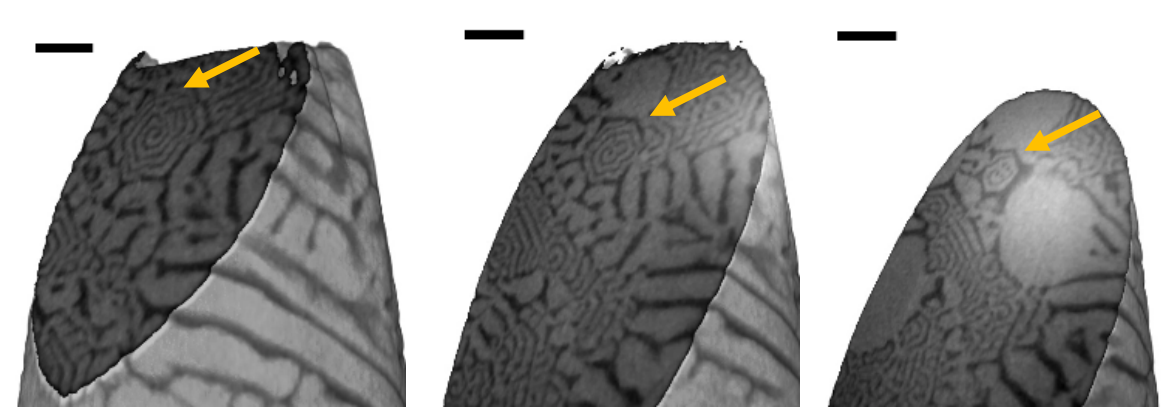

Figure 2. Reconstructions from ex situ $n \mathrm{TXM}$ on a Zeiss Xradia Ultra 800 X-ray microscope. Spiral $\mathrm{Zn}-\mathrm{MgZn}_{2}$ eutectic exhibits pyramidal enveloping shape. The panels are taken along the height of the spiral (left: top, middle: center, right: bottom). The arrows point to the same spiraling eutectic colony. The light gray phase is $\mathrm{Zn}$; the dark phase is $\mathrm{MgZn}_{2}$. Scale bars: $10 \mu \mathrm{m}$. 